\title{
Preoperative Prediction of Microvascular Invasion in Hepatocellular Carcinoma using Quantitative Image Analysis
}

\author{
Jian Zheng, MD ${ }^{1}$, Jayasree Chakraborty, PhD ${ }^{1}$, William C Chapman, MD, FACS ${ }^{2}$, Scott \\ Gerst, $\mathbf{M D}^{3}$, Mithat Gonen, PhD $^{4}$, Linda M Pak, MD ${ }^{1}$, William R Jarnagin, MD, FACS ${ }^{1}$, Ronald \\ P DeMatteo, MD, FACS ${ }^{1}$, Richard KG Do, MD, PhD ${ }^{3}$, and Amber L Simpson, PhD ${ }^{1}$ \\ Hepatopancreatobiliary Service in the Department of Surgery of the Memorial Sloan Kettering \\ Cancer Center, Research Staff in the Department of Surgery at Washington University School of \\ Medicine
}

\begin{abstract}
Background-Microvascular invasion (MVI) is a significant risk factor for early recurrence after resection or transplantation for hepatocellular carcinoma (HCC). Knowledge of MVI status would help guide treatment recommendations but is generally identified after surgery. This study aims to predict MVI preoperatively using quantitative image analysis.
\end{abstract}

Study Design-From 2 institutions, 120 patients submitted to resection of HCC from 2003 to 2015 were included. The largest tumor from preoperative CT was subjected to quantitative image analysis, which uses an automated computer algorithm to capture regional variation in CT enhancement patterns. Quantitative imaging features by automatic analysis, qualitative radiographic descriptors by 2 radiologists, and preoperative clinical variables were included in multivariate analysis to predict histologic MVI.

Results-Histologic MVI was identified in 19 (37\%) patients with tumors $5 \mathrm{~cm}$ and 34 (49\%) patients with tumors $>5 \mathrm{~cm}$. Among patients with $5 \mathrm{~cm}$ tumors, none of clinical findings or radiographic descriptors was associated with MVI; however, quantitative feature based on angle co-occurrence matrix predicted MVI with area under curve (AUC) 0.80 , positive predictive value

Correspondence address: Amber L. Simpson, PhD, Department of Surgery, Memorial Sloan Kettering Cancer Center, 1275 York Avenue, C-888, New York, NY 10065, Phone: 1 (212) 639-3624; Fax: 1 (917) 432-2387, simpsonl@mskcc.org.

${ }^{1}$ Department of Surgery, Memorial Sloan Kettering Cancer Center, New York, NY

2 Department of Surgery, Washington University School of Medicine, St. Louis, MO

${ }^{3}$ Department of Radiology, Memorial Sloan Kettering Cancer Center, New York, NY

${ }^{4}$ Department of Epidemiology and Biostatistics, Memorial Sloan Kettering Cancer Center, New York, NY

Members of the Hepatopancreatobiliary Service in the Department of Surgery and Memorial Sloan Kettering Cancer Center and Research Staff in the Department of Surgery at Washington University School of Medicine who co-authored this article are listed in the Appendix.

Disclosure Information: Nothing to disclose.

Disclosures outside the scope of this work: Dr Chapman is the Founder of Pathfinder Therapeutics and a member of the Advisory Board of Novartis.

An early draft of the technical methods was presented at the International Society for Optics and Photonics Medical Imaging Symposium, Orlando, FL, February 2017; and an abstract was presented at Americas Hepato-Pancreato-Biliary Association Annual Meeting, Miami Beach, FL, March 2017.

Publisher's Disclaimer: This is a PDF file of an unedited manuscript that has been accepted for publication. As a service to our customers we are providing this early version of the manuscript. The manuscript will undergo copyediting, typesetting, and review of the resulting proof before it is published in its final citable form. Please note that during the production process errors may be discovered which could affect the content, and all legal disclaimers that apply to the journal pertain. 
(PPV) $63 \%$ and negative predictive value (NPV) $85 \%$. In patients with $>5 \mathrm{~cm}$ tumors, higher afetoprotein (AFP) level, larger tumor size, and viral hepatitis history were associated with MVI, whereas radiographic descriptors did not. However, a multivariate model combining AFP, tumor size, hepatitis status, and quantitative feature based on local binary pattern predicted MVI with AUC 0.88, PPV 72\% and NPV 96\%.

Conclusions-This study reveals the potential importance of quantitative image analysis as a predictor of MVI.

\section{Keywords}

microvascular invasion; hepatocellular carcinoma; quantitative image analysis; image feature

\section{INTRODUCTION}

Hepatocellular carcinoma (HCC) is the most common liver cancer and its incidence is on the rise. ${ }^{1,2}$ Liver transplantation and resection are potentially curative for selected patients, but early recurrence is common, likely related to microvascular invasion (MVI) ${ }^{3-5} \mathrm{MVI}$ is an invasion of tumor cells into small vessels, which is associated with tumor dissemination and aggressive tumor biology. ${ }^{6-8}$ MVI can only be diagnosed reliably in postoperative specimens and is not readily discernible on preoperative imaging. ${ }^{9}$

It is important to detect MVI prior to surgery, as presence of MVI may discourage transplantation in favor of resection given the shortage of liver graft and thus the need to optimize the resource for those with low risk of recurrence ${ }^{5}, 10$. On the other hand, it would also be clinically relevant to predict absence of MVI, for both small tumors as well as for larger tumors that are within the extended criteria for transplantation, as the results may favor transplantation over resection ${ }^{11,12}$.

Preoperative biopsy may identify MVI but is useful only if positive; a negative result is not exclusionary due to tumor heterogeneity and sampling error. Furthermore, biopsy is not routinely performed prior to transplantation because of the risk of tumor seeding. $6,13,14$ Thus, many groups have studied noninvasive clinical predictors of MVI: larger tumor diameter, presence of multiple nodules, high level of a-fetoprotein (AFP), and thrombocytopenia. ${ }^{8,15-18}$ However, a nomogram based on some of these clinical predictors only predicted MVI with 57\% positive predictive value (PPV) in resected tumors within Milan criteria. ${ }^{15}$ In addition to clinical predictors of MVI, identification of MVI from preoperative computed tomography (CT) images has been attempted. Qualitative radiographic descriptors such as nonsmooth tumor margin and peritumoral enhancement have some association with MVI. ${ }^{9,} 19$ Other groups have also incorporated genomic and pathologic data with radiographic descriptors to develop radiogenomic venous invasion (RVI), an algorithm of three image traits that correlated with MVI. ${ }^{14,}{ }^{20}$ RVI was defined by the presence or absence of radiographic descriptors: presence of internal arteries, absence of a hypodense halo, and absence of tumor-liver difference. ${ }^{14,20}$ However, such qualitative radiographic descriptors depend on subjective evaluation by individual radiologists and are not validated in external cohorts. As shown in a repeatability study of major diagnostic 
scoring descriptors for HCC, even evaluation of classic descriptors of HCC such as washout appearance and pseudocapsule yield only moderate interobserver agreement. ${ }^{21}$

Radiomics is an emerging field that aims to quantify tumor heterogeneity related to changes in cellularity, necrosis, angiogenesis, and extracellular matrix deposition in tumor microenvironments. ${ }^{22,23}$ Quantitative image analysis is an automated computer algorithm to objectively quantify image heterogeneity by measuring spatial variations in pixel intensities. ${ }^{22,} 24$ The utility of quantitative image analysis for diagnosis and prognosis in cancer patients has been shown in prior studies, but it has never been previously explored to predict MVI. ${ }^{25-27}$ In this study, we aim to use preoperative CT quantitative image analysis to objectively identify predictors of MVI in resected HCC tumors, and also evaluate and compare to other previously reported predictors of MVI using clinical findings and subjective radiographic descriptors.

\section{METHODS}

\section{Patient selection}

With the approval of the institutional review boards of Memorial Sloan Kettering Cancer Center (MSKCC) and Washington University School of Medicine, we retrospectively analyzed patients who underwent resection of HCC with curative intent from 2003 to 2015 $(\mathrm{n}=348)$. At both institutions, patients with limited hepatic disease were considered for resection if they had resectable tumors, adequate future liver remnant for metabolic function, and absence of distant metastasis. $5,28,29$

All patients included in the study had abdominal CT within 3 months of surgery, with routine clinical imaging protocol for contrast-enhanced portal venous phase CT. Patients with no contrast-enhanced CT scan $(n=165)$ or with metal artifacts infiltrating the tumor on CT imaging $(n=5)$ were excluded. Patients treated previously with ablation, embolization, resection or transplant $(n=39)$ were excluded, as these treatments would alter the appearance of HCC on imaging and compromise the quantitative image analysis. Tumors that were ruptured $(n=6)$ or had a diffuse infiltrative pattern $(n=4)$ were also excluded since tumor borders were challenging to determine for analysis. Patients with gross macrovascular invasion $(n=9)$ were excluded. A total of 120 patients remained for inclusion in the study.

\section{Clinical and pathological variables}

Clinical and pathological variables were retrospectively reviewed. The variables analyzed included age at resection, gender, underlying liver disease, body mass index (BMI), Child Pugh classification, model for end-stage liver disease (MELD), a-fetoprotein (AFP), and platelet level. Operative details included operative blood loss and the number of segments resected. Resection of $\geq 3$ Couinaud segments was considered a major hepatectomy. Pathological data included number of tumors and satellites, largest tumor diameter, tumor cell differentiation, degree of tumor necrosis, hepatic capsule invasion, and margin status. The presence or absence of MVI on histology of the largest tumor, corresponding to the CT image, was also documented from the retrospective review. The grade of fibrosis and steatosis on adjacent liver was also recorded. 


\section{Qualitative radiographic descriptors}

Two hepatobiliary radiologists blinded to clinicopathological variables independently reviewed the CT scans and graded radiographic findings including number of tumors, largest tumor diameter, presence or absence of tumor internal arteries, hypodense halo, tumor-liver difference, nonsmooth margin, peripheral rim enhancement, and peritumoral enhancement as previously described. $9,14,19,20,30$

\section{Quantitative image analysis}

Automated quantitative image analysis was performed at the tumor periphery and the adjacent liver parenchyma motivated by qualitative radiographic descriptors such as hypodense halo and tumor-liver difference at the peritumoral region associated with MVI gene signatures ${ }^{14,20}$. This region of interest is termed the liver-tumor interface, which was defined as a 5 pixel-wide band inside and outside the tumor margin (Fig. 1A). The largest tumor and liver were segmented from CT using semi-automatic software (Scout Liver, Analogic Corporation, Peabody, MA, USA).

Quantitative image texture analysis was used to quantify variation in CT enhancement at the liver-tumor interface. Briefly, texture analysis captures differences in attenuation values in the scan using well-established techniques from the image analysis literature. Two types of texture features were studied: angle co-occurrence matrices (ACM) and local binary patterns (LBP). ACM quantifies the orientation patterns of neighboring pixels over a specified distance and direction ${ }^{31-33}$, whereas LBP quantifies the intensity patterns of the neighboring pixels $^{34-36}$. As previously described, 38 ACM and 128 LBP features were extracted for analysis. ${ }^{31,36}$ These features were evaluated at the liver-tumor interface, where 5 pixel-wide band inside the tumor margin was subtracted from outside the tumor margin. Quantitative image analysis was implemented in Matlab (Mathworks, Natick, MA, USA).

\section{Statistical analysis}

Significance of association between MVI and clinical variables was assessed using Fisher's exact test for categorical variables and Mann-Whitney for continuous variables. The unknown AFP levels for $9(8 \%)$ patients were imputed with other preoperative data using multivariate imputation by chained equations. ${ }^{37}$ For radiographic descriptors, Cohen's kappa statistic was used to quantify interobserver agreement. Significance of association between MVI and the radiographic descriptors was performed using Chi-squared test. Significance of association between MVI and quantitative imaging (texture) features was assessed with logistic regression. All preoperatively available variables (clinical, qualitative radiographic descriptor, and quantitative imaging features) significant on univariate analysis were including the multivariate analysis using logistic regression.

We created two multivariate models where the tumor was stratified by size, $5 \mathrm{~cm}$ and $>5$ $\mathrm{cm}$ to parallel stratification by Milan criteria and because greater heterogeneity in larger tumors may be related to intratumoral changes from angiogenesis, cell proliferation, and necrosis. ${ }^{22}$ The performance of each model as well as the overall performance of the combined models was evaluated using area under the receiver operating characteristic curve (AUC). This method evaluates a marker without the need for dichotomization, resulting in 
the receiver operating characteristic (ROC) curve, which provides an overall assessment of the predictive value of the marker. However, to make clinically relevant decisions, thresholds are needed to dichotomize the marker. These can be chosen in a variety of ways. The first threshold was selected to maximize sensitivity and specificity [maximizing the summation of sensitivity and specificity (Youden index)] to balance false positives and negatives. Given scarce organ availability, predicting absence of MVI may be more clinically relevant with improving allocation to those who have less aggressive tumor biology and benefit most from liver transplantation. Therefore, we evaluated the performance of our model with a second threshold which optimizes negative predictive value (NPV).

Analyses were performed using GraphPad Prism (GraphPad Software, CA) and Matlab (Mathworks, Natick, MA, USA). P value $<0.05$ from two-sided tests was considered statistically significant.

\section{RESULTS}

During 2003 to 2015, 120 patients submitted to resection of HCC were included in the study, with 104 patients from MSKCC and 16 patients from Washington University School of Medicine met the inclusion and exclusion criteria. A total of 53 (44\%) patients had tumors with pathologically confirmed MVI. Nineteen of 51 patients (37\%) with $5 \mathrm{~cm}$ tumors had MVI, and 34 of 69 patients (49\%) with $>5 \mathrm{~cm}$ tumors had MVI (Fig. 1B). The incidence of MVI was not statistically different between the two groups ( $\mathrm{p}=0.200$ ). Presence of MVI was associated with worse RFS among patients with tumors $5 \mathrm{~cm}$ (median RFS=16 vs 51 months, $\mathrm{p}=0.002$ ) and tumors $>5 \mathrm{~cm}$ (median RFS=12 vs 46 months, $\mathrm{p}=0.010$ ).

Clinical and pathological factors of both groups are shown (Table 1). In univariate analysis, no clinical variable was significantly associated with presence of MVI in patients with $\mathbf{5}$ $\mathrm{cm}$ tumors, although there was a non-significant AFP elevation in patients with MVI. By contrast, among tumors $>5 \mathrm{~cm}$, higher levels of AFP, larger tumor size, poor tumor differentiation, and history of viral hepatitis were all associated with presence of MVI. In the entire study population, larger tumor size on preoperative $\mathrm{CT}$ was associated with presence of MVI (median size $5.1 \mathrm{~cm}$ vs $8.3 \mathrm{~cm}$ for absence vs presence of MVI, respectively, $\mathrm{p}=0.006)$.

\section{Qualitative radiographic descriptors}

Two radiologists independently evaluated the largest tumor on preoperative CT of each patient for the presence or absence of tumor internal arteries, hypodense halo, tumor-liver difference, non-smooth margin, peripheral rim enhancement, and peritumoral enhancement. The ability of each radiologist to predict MVI as well as their interobserver agreement for all tumors, tumors $5 \mathrm{~cm}$, and tumors $>5 \mathrm{~cm}$ were analyzed (eTable 1). Only radiologist 1 was able to associate the presence of internal arteries and the absence of tumor-liver difference with MVI ( $\mathrm{p}=0.007$ and $\mathrm{p}=0.017$, respectively) in all tumors, but the level of agreement between the radiologists for all of the variables was modest, at best (kappa $=0.075$ and 0.452 , respectively). Since qualitative radiographic descriptors were not reliable predictors of MVI, these were not included in multivariate analysis. 


\section{Quantitative image features}

Of the total $38 \mathrm{ACM}$ and $128 \mathrm{LBP}$ features evaluated, $16 \mathrm{ACM}$ and $21 \mathrm{LBP}$ features were significantly associated with MVI in tumors $5 \mathrm{~cm}$ and $>5 \mathrm{~cm}$, respectively, on univariate analysis. For simplicity and to avoid overloading the prediction model with quantitative features, the most significant single feature by univariate analysis was included in multivariate analysis using logistic regression.

\section{Multivariate preoperative MVI prediction model}

We created two multivariate models where the tumor was stratified by size, $5 \mathrm{~cm}$ and $>5$ $\mathrm{cm}$, to align with Milan criteria, and we also presented an overall performance of combined model. Among patients with $5 \mathrm{~cm}$ tumors, only AFP approached significance on univariate analysis of all preoperatively available clinical variables and was therefore included in the multivariate model. A single ACM feature in the tumor-liver interface predicted MVI with AUC 0.80 . A positive predictive value (PPV) of $63 \%$ and negative predictive value (NPV) of $85 \%$ were achieved when both sensitivity and specificity were optimized, which were $79 \%$ and $72 \%$, respectively (Fig. 2). When a threshold was applied to achieve $100 \%$ NPV, to predict absence of MVI in order to limit the rate of postoperative recurrence and thus to provide greater benefit from transplant, the model yielded $100 \%$ sensitivity, with corresponding decreases in PPV and specificity. The performance of prediction model did not improve with the addition of AFP.

In patients with $>5 \mathrm{~cm}$ tumors, a single local binary pattern (LBP) feature in the tumor-liver interface alone predicted MVI with AUC 0.75. A PPV of $83 \%$ and NPV of $69 \%$ were obtained when both sensitivity and specificity were optimized (Fig. 3). In this group, higher level of AFP, larger tumor size, and history of viral hepatitis were associated with MVI in the univariate analysis. Thus, these preoperative clinical variables were added to the LBP feature in multivariate analysis, which yielded AUC 0.88, PPV 72\% and NPV 96\%. Again, the prediction model can obtain 100\% NPV (identify absence of MVI in tumors $>5 \mathrm{~cm}$ that may still be favorable for transplant) and $100 \%$ sensitivity, with corresponding decreases in PPV and specificity.

The overall performance of the proposed models for all patients obtained an AUC of 0.85 . Since for tumors $5 \mathrm{~cm}$ the model with a single ACM feature provides the best performance, and for tumors $>5 \mathrm{~cm}$ the model with a single LBP, AFP, largest tumor size, and hepatitis provides the best performance, we considered these variables in overall performance. This method achieved the optimal sensitivity and specificity of $89 \%$ and $67 \%$ with a PPV and NPV of $68 \%$ and $91 \%$ while balancing sensitivity and specificity. The NPV of $100 \%$ is achieved with a sensitivity of $100 \%$ and specificity and NPV of $40 \%$ and 57\%, respectively.

\section{DISCUSSION}

In this study, we show that quantitative image analysis is a promising preoperative predictor of MVI in patients with HCC. Quantitative features alone were powerful predictors, offering a NPV of $85 \%$ among $5 \mathrm{~cm}$ tumors and $69 \%$ among $>5 \mathrm{~cm}$ tumors, and the NPV can also be optimized to yield $100 \%$ NPV for tumors of all sizes. This is clinically significant 
because predicting absence of MVI among HCC tumors with high NPV may favor liver transplantation over resection. On the other hand, the ability to predict the presence of MVI with high PPV may discourage transplantation in favor of other treatments.

The critical shortage of available organs and long waiting lists mandates the most effective and rational use of this scarce resource. To offer the best outcome, liver transplantation is generally limited to patients with tumors that meet Milan criteria, which includes either a single tumor that is $\leq 5 \mathrm{~cm}$ or up to 3 tumors that are $\leq 3 \mathrm{~cm}$ each, to minimize the risk of recurrence. ${ }^{38,39}$ Several groups have challenged Milan criteria and called for expanded criteria, including use of $\$ 8 \mathrm{~cm}$ total tumor diameter as proposed by University of California, San Francisco. ${ }^{11,12}$ These clinically based classification schemes have proven useful for risk stratification but are clearly imperfect, since many patients within the criteria have aggressive tumors that should not be considered for transplantation, and likewise some patients with disease outside the criteria have favorable biology and would potentially benefit from this therapy. In this regard, the use of quantitative image analysis to predict for MVI may serve as an important adjunct variable to the extended criteria and further optimize patient selection for liver transplantation. For example, tumors outside of Milan criteria (i.e. $>5 \mathrm{~cm}$ ) but without MVI may indicate a less aggressive disease biology that may benefit from transplantation. On the other hand, patients with small tumors but with evidence of MVI with a high PPV may be more suitable for other approaches. This would be especially prudent in patients with high operative risk from significant co-morbidities. If patients were known to have high risk of MVI and consequent recurrence, perhaps they may be better treated with upfront transarterial chemoembolization followed by resection at a later time if no new disease manifests.

Quantitative image analysis can predict MVI status among both $5 \mathrm{~cm}$ and $>5 \mathrm{~cm}$ HCC tumors, with best features derived from ACM and LBP, respectively. This difference warrants further prospective evaluation of the tumor specimen, but it is likely because smaller tumors appear more homogeneous on imaging, with outward growth of tumor best captured by directional changes in intensity as measured by ACM features. On the other hand, larger tumors appear more heterogeneous and thus best quantified by local variations in pixel intensity as in LBP analysis. Heterogeneous tumors may reflect underlying poor cell differentiation with areas of necrosis, angiogenesis, and extracellular matrix deposition. ${ }^{22}$

The most predictive feature came from the differences in pixel variations between the HCC near the tumor border and adjacent liver parenchyma. This finding was not unexpected given that even radiographic descriptors from the literature have similarly shown that the peritumoral region was the most informative region, including presence or absence of hypodense halo, tumor-liver difference, non-smooth margin, peripheral rim enhancement, and peritumoral enhancement. ${ }^{9}, 14,19,20,30,40$ This tumor-liver interface may reflect underlying tumor distortion of tissue from microvascular invasion, cellular proliferation, associated inflammatory response and extracellular matrix remodeling. ${ }^{14,20,41}$ The tumor edge has been shown in an experimental study to be a site of tumor-host crosstalk via signaling of transforming growth factor- $\beta$ and platelet-derived growth factor and thus crucial in tumor cell progression. ${ }^{42}$ 
In addition to quantitative image analysis, we also evaluated the predictive power of preoperative clinical variables. High levels of AFP, larger tumor size, and a history of chronic viral hepatitis were associated with MVI among tumors $>5 \mathrm{~cm}$, consistent with prior findings. ${ }^{8,15-18}$ AFP and tumor size among $5 \mathrm{~cm}$ tumors were not associated with MVI. This is likely because the rate of MVI among tumors $5 \mathrm{~cm}$ was relatively low at $37 \%$. Prior studies have also shown that tumors $\_\mathrm{cm}$ and $2-4 \mathrm{~cm}$ had significantly lower rate of MVI (25\% and $31 \%$, respectively) compared to tumors that reached $\geq 4 \mathrm{~cm}(50 \%) .{ }^{43}$ Since only 11 patients $(9 \%)$ had multiple tumors on preoperative CT, we did not have the statistical power to associate MVI with multinodularity. The addition of quantitative image features to preoperative clinical variables that were associated with MVI improved the prediction model. Radiographic descriptors were not included in the multivariate model because only radiologist 1 was able to significantly associate MVI with the presence of internal arteries and absence of tumor-liver difference, but the interobserver agreement with radiologist 2 were only slight and moderate.

This study's use of quantitative image analysis to predict MVI is novel and clinically important. Not only is imaging analysis noninvasive and suitable for use in serial follow-up CT scans, its sensitivity for MVI detection surpassed that of preoperative biopsy. ${ }^{14}$ In addition, quantitative image analysis is an unbiased and systematic method that can be used by other institutions. We have performed quantitative image analysis only on CT images, but its use on other imaging modalities, such as magnetic resonance imaging (MRI), to predict MVI has not been explored. A limitation of our study is the relatively small sample size, as many patients were excluded due to lack of adequate CT images and many patients only had MRI. Similar approaches could be developed for MRI scans. In addition, we did not include transplanted patients in this study. Many of the patients included had Child's Pugh class A and some with solitary or multinodular tumors within the Milan criteria and were eligible for both resection and transplantation. Thus, our result would be applicable to a subset of patients who are considered for transplantation, but this needs to be validated in a larger cohort of patients with advanced liver disease. We did not include transplant patients in this study primarily because majority of patients received pre-transplant locoregional therapies, which would alter the image characteristic of HCC. Furthermore, since CT scans were obtained up to 3 months preoperatively, it is possible for MVI to have developed after the scan and before resection in some cases, thereby reducing the predictive utility. However, the median time from CT to resection in this study was 20 days and thus new MVI is unlikely to a major source of error. We also did not examine tumor histology to correlate quantitative imaging features with the exact location(s) of MVI, but promising results from this study may guide future prospective studies with more comprehensive radio-pathologic correlations.

\section{CONCLUSION}

In summary, we showed for the first time that quantitative image analysis can predict MVI status among both $5 \mathrm{~cm}$ and $>5 \mathrm{~cm}$ HCC tumors and that the addition of clinical data improved the predictive model among larger tumors. External validation of quantitative image analysis for the prediction of MVI is warranted, as it may help guide treatment recommendations for patients considered for resection or liver transplantation. 


\section{Supplementary Material}

Refer to Web version on PubMed Central for supplementary material.

\section{Acknowledgments}

Support: This work was supported in part by NIH/NCI P30 CA008748 Cancer Center Support Grant.

$\begin{array}{ll}\text { Abbreviations } & \\ \text { ACM } & \text { angle co-occurrence matrices } \\ \text { AFP } & \text { a-fetoprotein } \\ \text { AUC } & \text { area under curve } \\ \text { BMI } & \text { body mass index } \\ \text { CT } & \text { computed tomography } \\ \text { HCC } & \text { hepatocellular carcinoma } \\ \text { LBP } & \text { local binary patterns } \\ \text { MELD } & \text { model for end-stage liver disease } \\ \text { MVI } & \text { microvascular invasion } \\ \text { NPV } & \text { negative predictive value } \\ \text { PPV } & \text { positive predictive value } \\ \text { RFS } & \text { recurrence-free survival } \\ \text { RVI } & \text { radiogenomic venous invasion } \\ \text { MRI } & \text { magnetic resonance imaging }\end{array}$

\section{References}

1. Forner A, Llovet JM, Bruix J. Hepatocellular carcinoma. Lancet. 2012; 379:1245-1255. [PubMed: 22353262]

2. Torre LA, Bray F, Siegel RL, et al. Global cancer statistics, 2012. CA Cancer J Clin. 2015; 65:87108. [PubMed: 25651787]

3. Hirokawa F, Hayashi M, Asakuma M, et al. Risk factors and patterns of early recurrence after curative hepatectomy for hepatocellular carcinoma. Surg Oncol. 2016; 25:24-29. [PubMed: 26979637]

4. Yamamoto Y, Ikoma H, Morimura R, et al. Optimal duration of the early and late recurrence of hepatocellular carcinoma after hepatectomy. World J Gastroenterol. 2015; 21:1207-1215. [PubMed: 25632194]

5. Chapman WC, Klintmalm G, Hemming A, et al. Surgical treatment of hepatocellular carcinoma in North America: can hepatic resection still be justified? J Am Coll Surg. 2015; 220:628-637. [PubMed: 25728142] 
6. Rodriguez-Peralvarez M, Luong TV, Andreana L, et al. A systematic review of microvascular invasion in hepatocellular carcinoma: diagnostic and prognostic variability. Ann Surg Oncol. 2013; 20:325-339. [PubMed: 23149850]

7. Sumie S, Nakashima O, Okuda K, et al. The significance of classifying microvascular invasion in patients with hepatocellular carcinoma. Ann Surg Oncol. 2014; 21:1002-1009. [PubMed: 24254204]

8. Zhao WC, Fan LF, Yang N, et al. Preoperative predictors of microvascular invasion in multinodular hepatocellular carcinoma. Eur J Surg Oncol. 2013; 39:858-864. [PubMed: 23669199]

9. Renzulli M, Brocchi S, Cucchetti A, et al. Can current preoperative imaging be used to detect microvascular invasion of hepatocellular carcinoma? Radiology. 2016; 279:432-442. [PubMed: 26653683]

10. Dhir M, Melin AA, Douaiher J, et al. A review and update of treatment options and controversies in the management of hepatocellular carcinoma. Ann Surg. 2016; 263:1112-1125. [PubMed: 26813914]

11. Yao FY, Xiao L, Bass NM, et al. Liver transplantation for hepatocellular carcinoma: validation of the UCSF-expanded criteria based on preoperative imaging. Am J Transplant. 2007; 7:2587-2596. [PubMed: 17868066]

12. Zaydfudim VM, Vachharajani N, Klintmalm GB, et al. Liver resection and transplantation for patients with hepatocellular carcinoma beyond Milan Criteria. Ann Surg. 2016; 264:650-658. [PubMed: 27433910]

13. Pawlik TM, Gleisner AL, Anders RA, et al. Preoperative assessment of hepatocellular carcinoma tumor grade using needle biopsy: implications for transplant eligibility. Ann Surg. 2007; 245:435442. [PubMed: 17435551]

14. Banerjee S, Wang DS, Kim HJ, et al. A computed tomography radiogenomic biomarker predicts microvascular invasion and clinical outcomes in hepatocellular carcinoma. Hepatology. 2015; 62:792-800. [PubMed: 25930992]

15. Lei Z, Li J, Wu D, et al. Nomogram for preoperative estimation of microvascular invasion risk in hepatitis B virus-related hepatocellular carcinoma within the Milan Criteria. JAMA Surg. 2016; 151:356-363. [PubMed: 26579636]

16. Schlichtemeier SM, Pang TC, Williams NE, et al. A pre-operative clinical model to predict microvascular invasion and long-term outcome after resection of hepatocellular cancer: The Australian experience. Eur J Surg Oncol. 2016; 42:1576-1583. [PubMed: 27378158]

17. Kaibori M, Ishizaki M, Matsui K, et al. Predictors of microvascular invasion before hepatectomy for hepatocellular carcinoma. J Surg Oncol. 2010; 102:462-468. [PubMed: 20872949]

18. Sumie S, Kuromatsu R, Okuda K, et al. Microvascular invasion in patients with hepatocellular carcinoma and its predictable clinicopathological factors. Ann Surg Oncol. 2008; 15:1375-1382. [PubMed: 18324443]

19. Chou CT, Chen RC, Lin WC, et al. Prediction of microvascular invasion of hepatocellular carcinoma: preoperative CT and histopathologic correlation. AJR Am J Roentgenol. 2014; 203:W253-259. [PubMed: 25148181]

20. Segal E, Sirlin CB, Ooi C, et al. Decoding global gene expression programs in liver cancer by noninvasive imaging. Nat Biotechnol. 2007; 25:675-680. [PubMed: 17515910]

21. Davenport MS, Khalatbari S, Liu PS, et al. Repeatability of diagnostic features and scoring systems for hepatocellular carcinoma by using MR imaging. Radiology. 2014; 272:132-142. [PubMed: 24555636]

22. Davnall F, Yip CS, Ljungqvist G, et al. Assessment of tumor heterogeneity: an emerging imaging tool for clinical practice? Insights Imaging. 2012; 3:573-589. [PubMed: 23093486]

23. Aerts HJ, Velazquez ER, Leijenaar RT, et al. Decoding tumour phenotype by noninvasive imaging using a quantitative radiomics approach. Nat Commun. 2014; 5:4006. [PubMed: 24892406]

24. Simpson AL, Adams LB, Allen PJ, et al. Texture analysis of preoperative CT images for prediction of postoperative hepatic insufficiency: a preliminary study. J Am Coll Surg. 2015; 220:339-346. [PubMed: 25537305]

25. Li M, Fu S, Zhu Y, et al. Computed tomography texture analysis to facilitate therapeutic decision making in hepatocellular carcinoma. Oncotarget. 2016; 7:13248-13259. [PubMed: 26910890] 
26. Huang YL, Chen JH, Shen WC. Diagnosis of hepatic tumors with texture analysis in nonenhanced computed tomography images. Acad Radiol. 2006; 13:713-720. [PubMed: 16679273]

27. Raman SP, Schroeder JL, Huang P, et al. Preliminary data using computed tomography texture analysis for the classification of hypervascular liver lesions: generation of a predictive model on the basis of quantitative spatial frequency measurements--a work in progress. J Comput Assist Tomogr. 2015; 39:383-395. [PubMed: 25700222]

28. Ribero D, Curley SA, Imamura H, et al. Selection for resection of hepatocellular carcinoma and surgical strategy: indications for resection, evaluation of liver function, portal vein embolization, and resection. Ann Surg Oncol. 2008; 15:986-992. [PubMed: 18236112]

29. Dhir M, Melin AA, Douaiher J, et al. A review and update of treatment options and controversies in the management of hepatocellular carcinoma. Ann Surg. 2016

30. An C, Kim DW, Park YN, et al. Single hepatocellular carcinoma: preoperative mr imaging to predict early recurrence after curative resection. Radiology. 2015; 276:433-443. [PubMed: 25751229]

31. Chakraborty J, Rangayyan RM, Banik S, et al. Detection of architectural distortion in prior mammograms using statistical measures of orientation of texture. Medical Imaging: 2012 Computer-Aided Diagnosis. 2012:8315.

32. Chakraborty J, Rangayyan RM, Banik S, et al. Statistical measures of orientation of texture for the detection of architectural distortion in prior mammograms of interval-cancer. J Electronic Imaging. 2012; 21(3)

33. Chakraborty J, Midya A, Mukhopadhyay S, et al. Automatic Characterization of Masses in Mammograms. Proceedings of the 2013 6th International Conference on Biomedical Engineering and Informatics (Bmei 2013). 2013; 1 and 2:111-115.

34. Ojala T, Pietikainen M, Maenpaa T. Multiresolution gray-scale and rotation invariant texture classification with local binary patterns. IEEE Transactions on Pattern Analysis and Machine Intelligence. 2002; 24:971-987.

35. Alic L, Niessen WJ, Veenland JF. Quantification of heterogeneity as a biomarker in tumor imaging: a systematic review. PLoS One. 2014; 9:e110300. [PubMed: 25330171]

36. Chakraborty J, Langdon-Embry L, Escalon JG, et al. Texture analysis for survival prediction of pancreatic ductal adenocarcinoma patients with neoadjuvant chemotherapy. Medical Imaging 2016: Image Processing. 2016:9784.

37. Buuren, Sv. Flexible Imputation of Missing Data. Chapman \& Hall/CRC Press; 2012.

38. Mazzaferro V, Bhoori S, Sposito C, et al. Milan criteria in liver transplantation for hepatocellular carcinoma: an evidence-based analysis of 15 years of experience. Liver Transpl. 2011; 17:S44-57. [PubMed: 21695773]

39. Mazzaferro V, Regalia E, Doci R, et al. Liver transplantation for the treatment of small hepatocellular carcinomas in patients with cirrhosis. N Engl J Med. 1996; 334:693-699. [PubMed: 8594428]

40. Ahn SY, Lee JM, Joo I, et al. Prediction of microvascular invasion of hepatocellular carcinoma using gadoxetic acid-enhanced MR and (18)F-FDG PET/CT. Abdom Imaging. 2015; 40:843-851. [PubMed: 25253426]

41. Unal E, Idilman IS, Akata D, et al. Microvascular invasion in hepatocellular carcinoma. Diagn Interv Radiol. 2016; 22:125-132. [PubMed: 26782155]

42. van Zijl F, Mair M, Csiszar A, et al. Hepatic tumor-stroma crosstalk guides epithelial to mesenchymal transition at the tumor edge. Oncogene. 2009; 28:4022-4033. [PubMed: 19718050]

43. Esnaola NF, Lauwers GY, Mirza NQ, et al. Predictors of microvascular invasion in patients with hepatocellular carcinoma who are candidates for orthotopic liver transplantation. J Gastrointest Surg. 2002; 6:224-232. discussion 232. [PubMed: 11992808]

\section{Appendix}

Members of the Hepatopancreatobiliary Service of the Department of Surgery and Memorial Sloan Kettering Cancer Center: Peter J Allen, MD, FACS, Vinod P Balachandran, MD, 
FACS, Michael I D'Angelica, MD, FACS, T Peter Kingham, MD, FACS. Members of the Research Staff of the Department of Surgery at Washington University School of Medicine: Neeta Vachharajani, BS. 

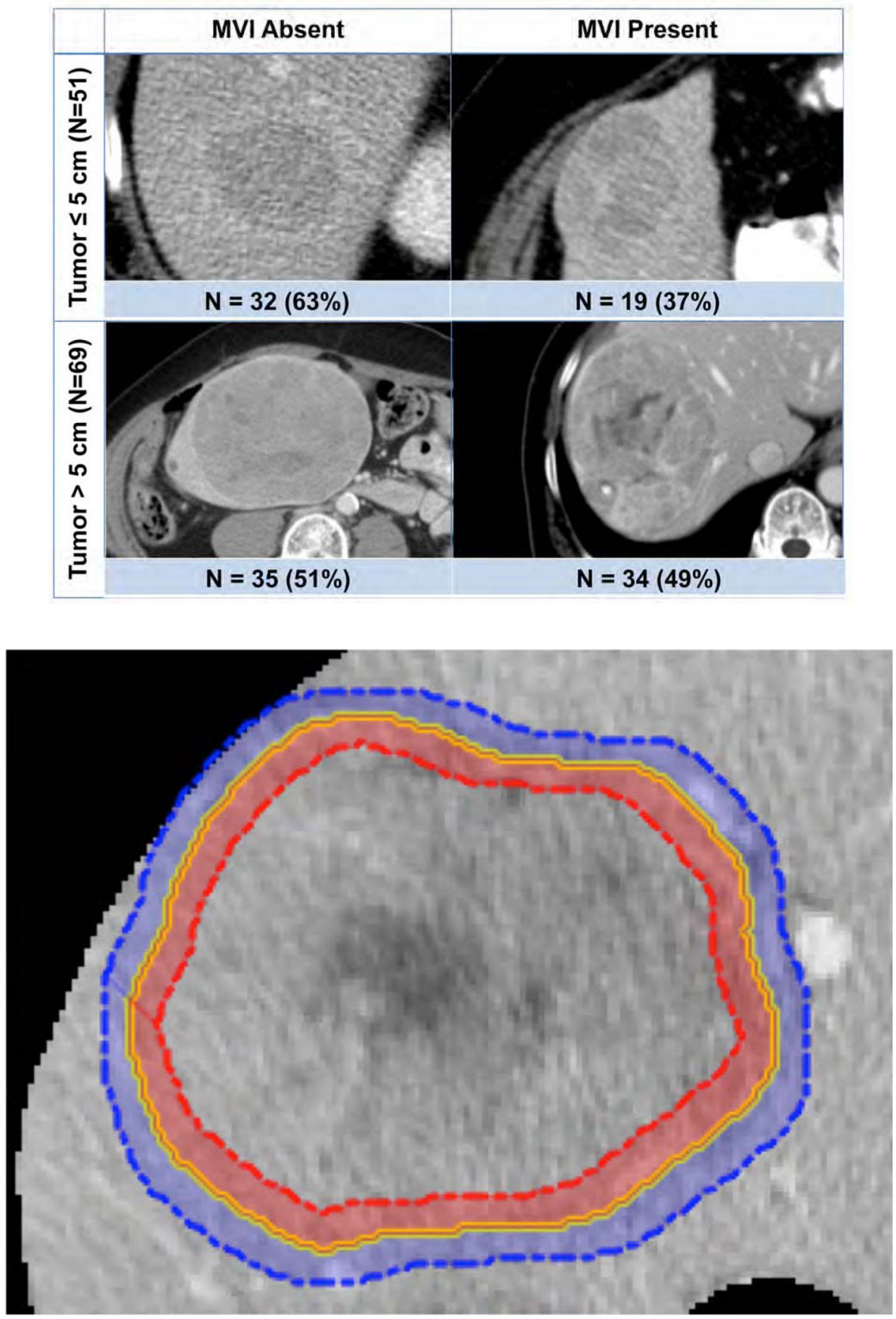

Figure 1.

(A) Quantitative image analysis was performed on tumor-liver interface, where 5 pixel-wide band inside the tumor margin (red shade on the tumor periphery) was subtracted from outside the tumor margin (blue shade on the adjacent hepatocytes). (B) The largest tumor from each patient was used to perform quantitative image analysis to predict microvascular invasion (MVI). 


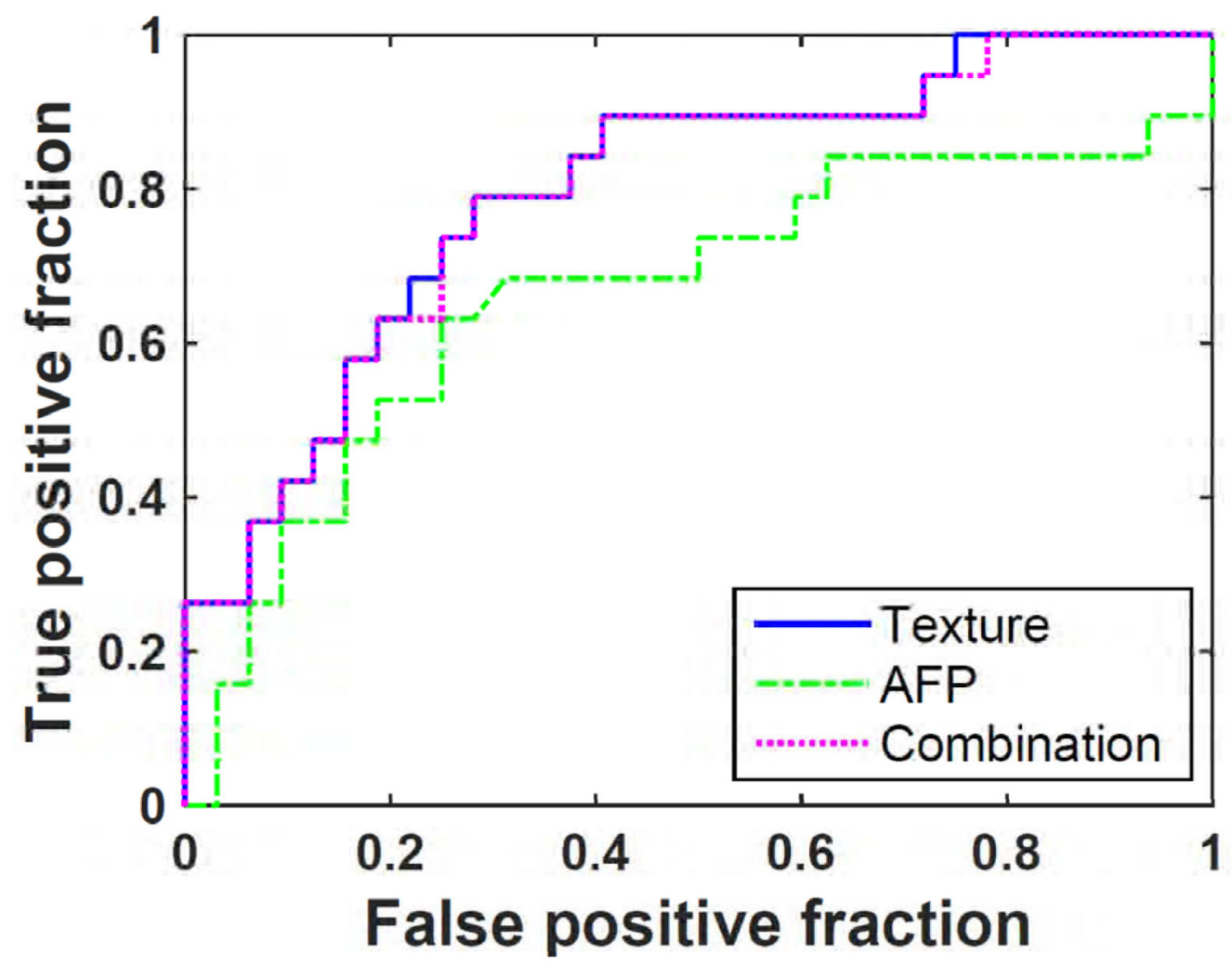

\begin{tabular}{|l|c|c|c|c|c|c|c|c|c|}
\hline Variables & AUC & \multicolumn{3}{|c|}{ Optimized sensitivity and specificity } & \multicolumn{4}{c|}{ Optimized NPV } \\
\cline { 3 - 10 } & $(95 \%$ CI) & Sensitivity & Specificity & PPV & NPV & Sensitivity & Specificity & PPV & NPV \\
\hline ACM & 0.80 & 0.79 & 0.72 & 0.63 & 0.85 & 1.00 & 0.25 & 0.44 & 1.00 \\
& $(0.65-0.90)$ & $(0.61-0.97)$ & $(0.56-0.88)$ & $(0.43-0.82)$ & $(0.44-0.81)$ & $(0.82-1.00)$ & $(0.12-0.43)$ & $(0.29-0.60)$ & $(0.63-1.00)$ \\
\hline AFP & 0.67 & 0.63 & 0.75 & 0.60 & 0.77 & 0.84 & 0.38 & 0.44 & 0.80 \\
& $(0.48-0.81)$ & $(0.38-0.84)$ & $(0.57-0.89)$ & $(0.36-0.81)$ & $(0.59-0.90)$ & $(0.60-0.97)$ & $(0.21-0.56)$ & $(0.28-0.62)$ & $(0.52-0.96)$ \\
\hline ACM + AFP & 0.79 & 0.79 & 0.72 & 0.63 & 0.85 & 1.00 & 0.22 & 0.43 & 1.00 \\
& $(0.64-0.90)$ & $(0.54-0.94)$ & $(0.53-0.86)$ & $(0.41-0.81)$ & $(0.66-0.96)$ & $(0.82-1.00)$ & $(0.09-0.40)$ & $(0.28-0.59)$ & $(0.59-1.00)$ \\
\hline
\end{tabular}

Figure 2.

Quantitative image analysis of angle co-occurrence matrix (ACM) predicted microvascular invasion among $5 \mathrm{~cm}$ tumors. ACM, angle co-occurrence matrix; AFP, a-fetoprotein; AUC, area under curve; CI, confidence interval; PPV, positive predictive value; NPV, negative predictive value. $95 \%$ confidence intervals are presented in parentheses. 

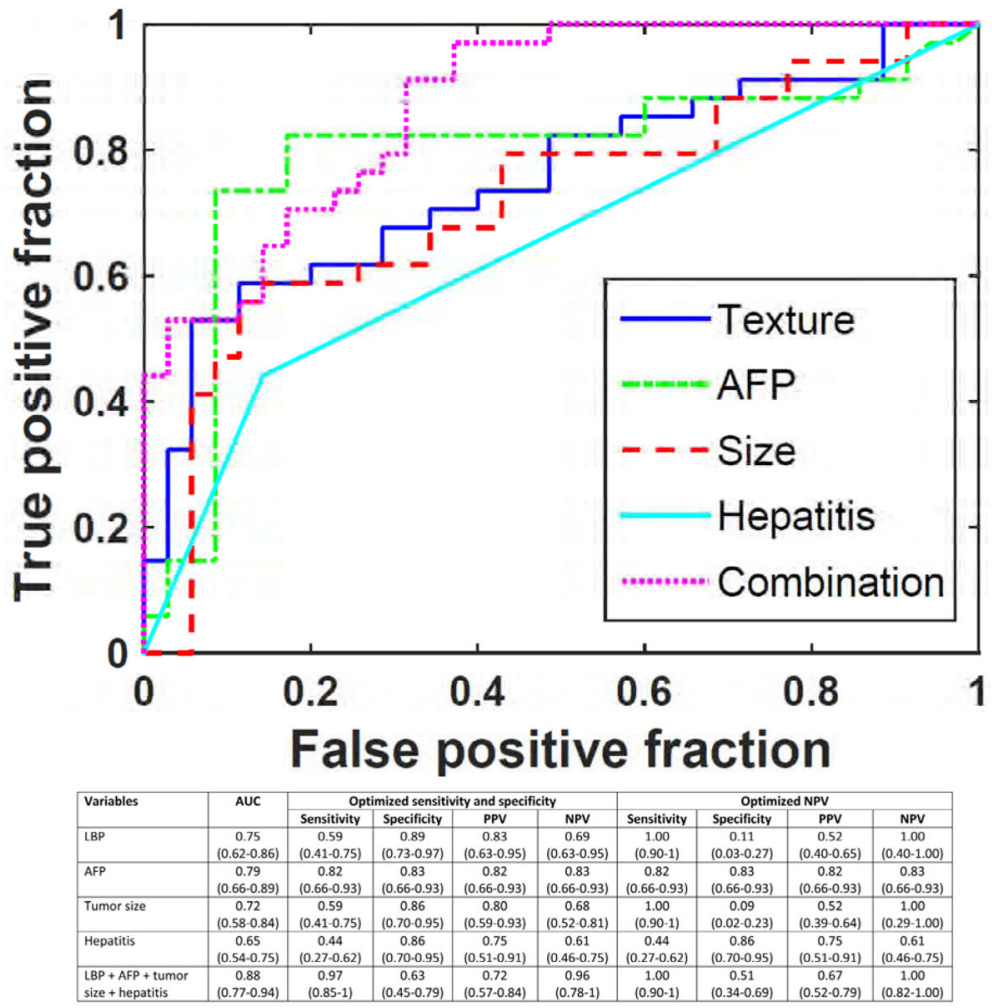

Figure 3.

Clinical and quantitative image analysis of local binary pattern (LBP) predicted microvascular invasion among $>5 \mathrm{~cm}$ tumors. LBP, local binary pattern; AFP, a-fetoprotein; AUC, area under curve; CI, confidence interval; PPV, positive predictive value, NPV, negative predictive value. $95 \%$ confidence intervals are presented in parentheses. 


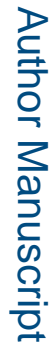

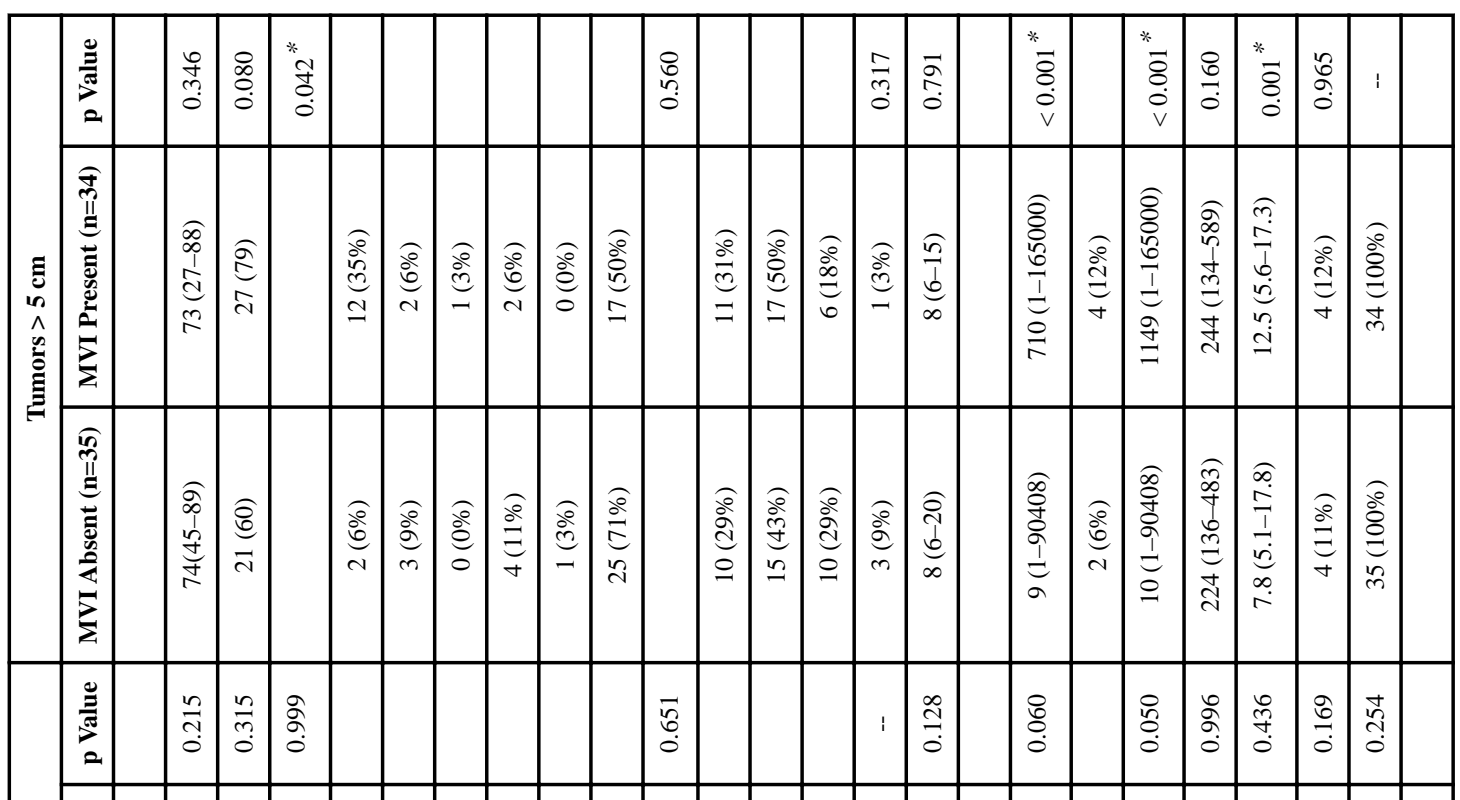

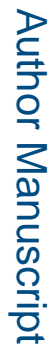

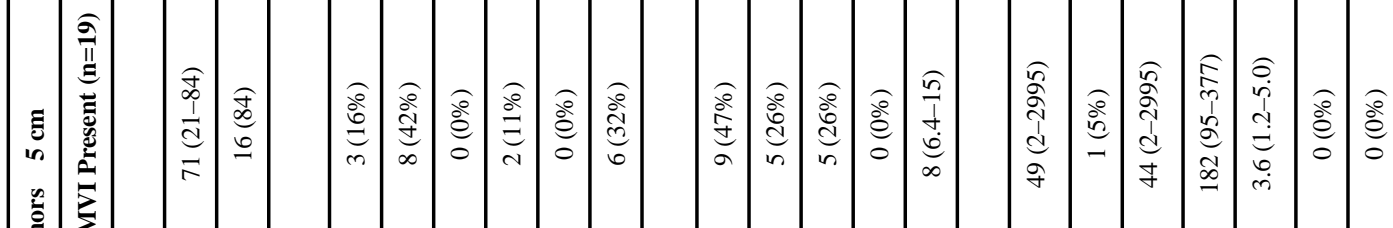

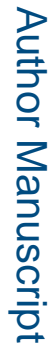

害

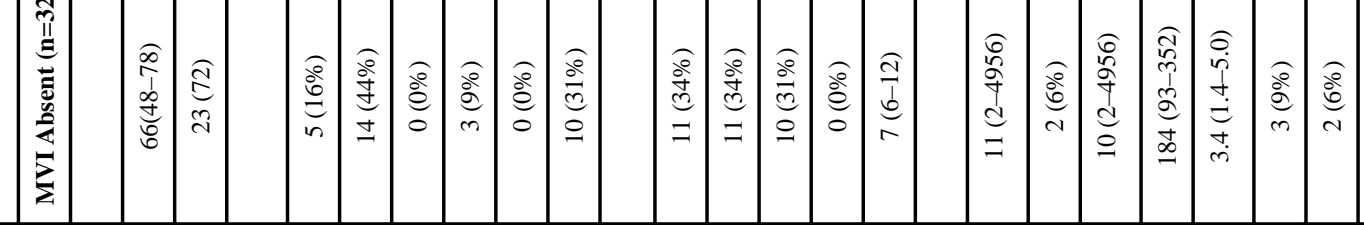

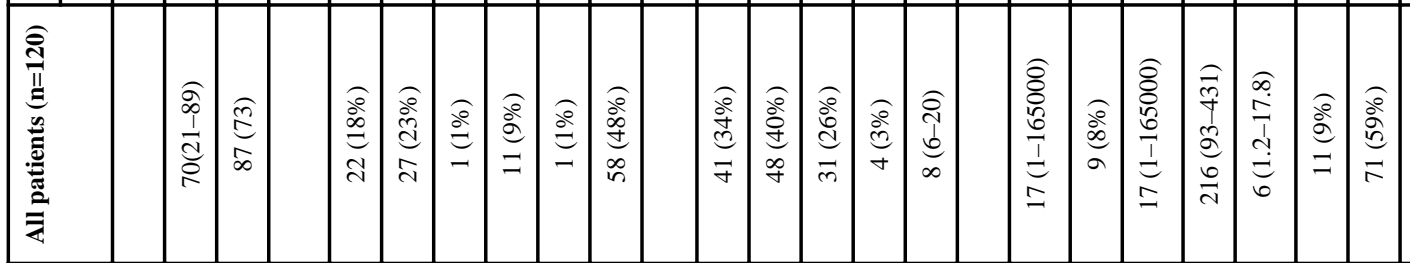

D

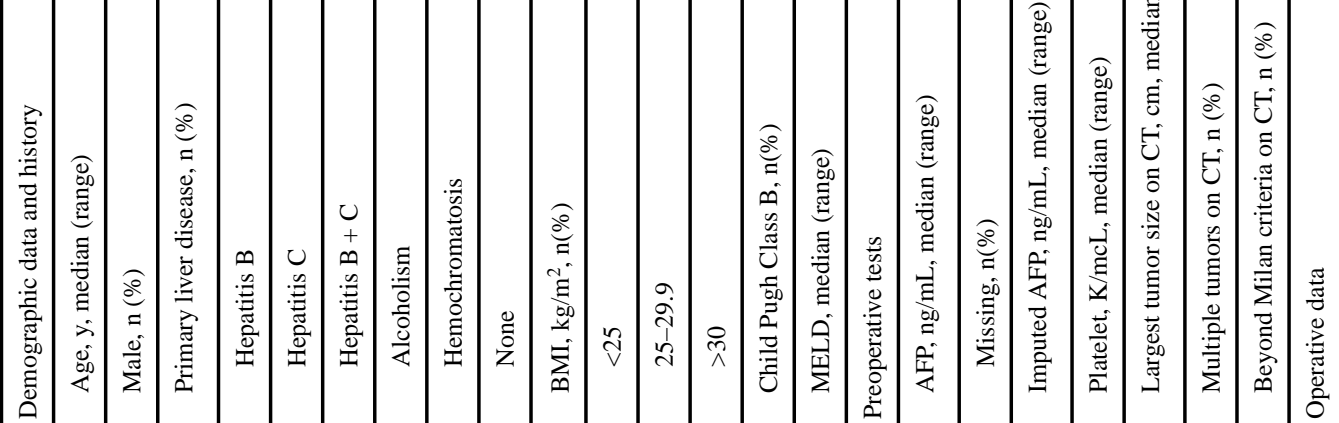

J Am Coll Surg. Author manuscript; available in PMC 2018 December 01. 


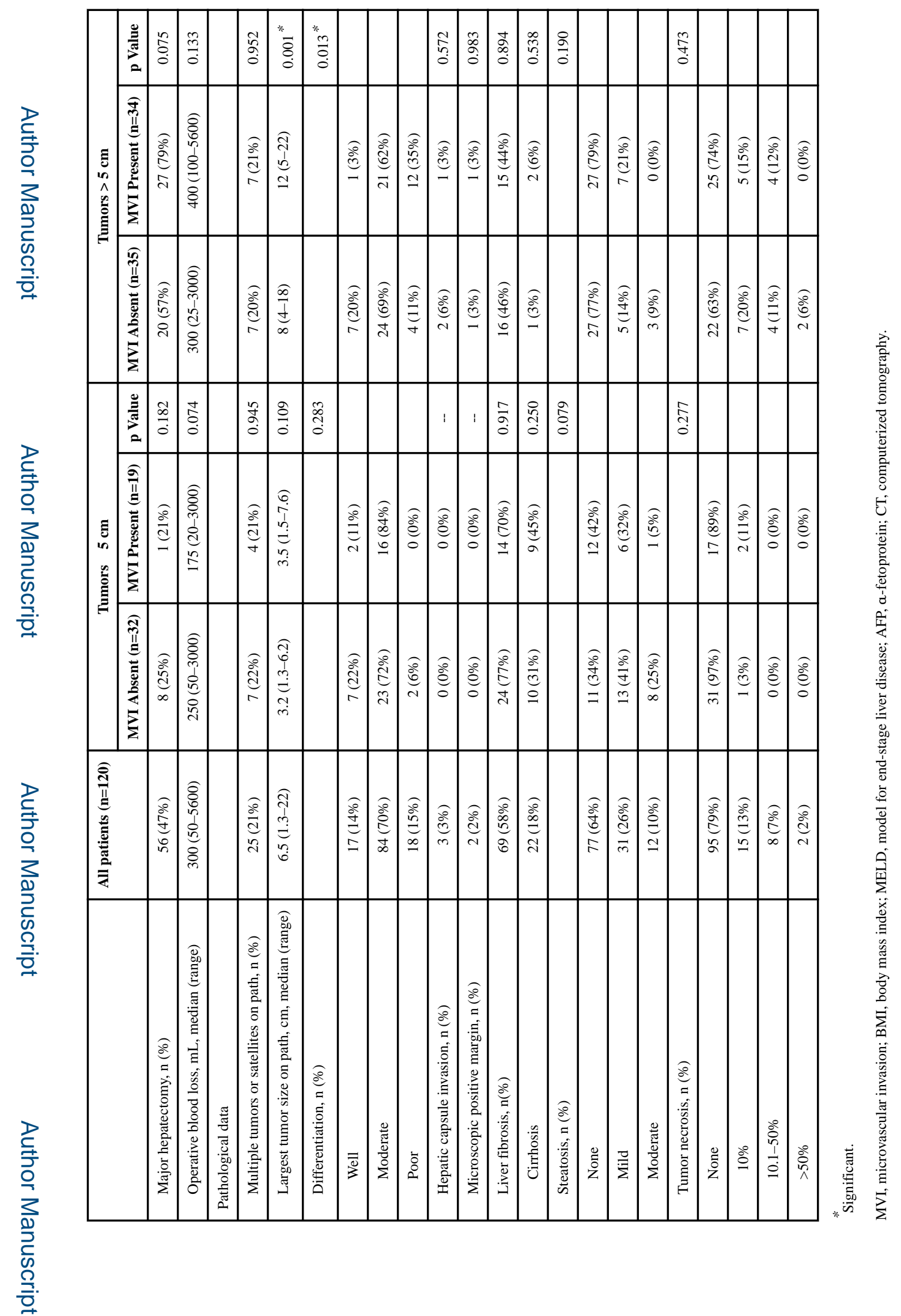

J Am Coll Surg. Author manuscript; available in PMC 2018 December 01. 